\title{
REA_14 - Developing a lab-in-a-box and low-cost paper-based sensors for ZIKV and CHIKV diagnosis in Latin America
}

Margot Karlikow ${ }^{1}$; Severino Jefferson Ribeiro da Silva ${ }^{2 *}$; Yuxiu Guo ${ }^{1}$; Seray Cicek ${ }^{1}$; Larissa Krokovsky²; Jim Collins ${ }^{3}$; Alexander Green ${ }^{4}$; Constância Ayres²; Lindomar Pena ${ }^{2}$; Keith Pardee ${ }^{1}$.

${ }^{1}$ University of Toronto;

${ }^{2}$ Fiocruz - Fundação Oswaldo Cruz;

${ }^{3}$ Harvard University;

${ }^{4}$ Arizona State University.

Introduction: Zika virus (ZIKV) has emerged as a major global public health concern in the last five years due to its link as a causative agent of congenital malformations in thousands of newborns. Currently, the reverse transcriptase reaction followed by quantitative polymerase chain reaction (RT-qPCR) is considered the reference method to diagnose ZIKV-infection. Nevertheless, RT-qPCR requires technical expertise and utilizes specialized equipment for amplification and detection of viral genome. These drawbacks negatively impact the establishment of effective disease control programs caused by ZIKV, especially in low-resource areas. This bottleneck in diagnostic capacity led to calls for molecular diagnostics that can be used at the point-of-care (POC).

Objective: In this context, the aim of this work was to develop low-cost paper-based sensors for ZIKV and CHIKV detection and a lab-in-a-box hardware to assist the visualization of results.

Methodology: Using computationally designed toehold switch-based sensors targeting the ZIKV and CHIKV genomes, we developed a paper-based test using cell-free protein expression reactions that could be freeze-dried for distribution without refrigeration. Containing the recombinant enzymes of transcription and translation from E. coli, these reactions first transcribe the RNA-based toehold switch from a DNA template and then, if the target ZIKV/CHIKV viral sequences are present, translate a reporter protein (e.g. LacZ) to create an optical signal (yellow to purple). Then, we developed a portable, high-capacity "lab-in-a-box" that serves as a low-cost, temperature-controlled plate reader ( $\sim 500$ USD). Finally, we validated the assay for ZIKV detection using 268 patient samples collected at the epicenter of the last Zika epidemic in Latin America. Given the success of the ZIKV validation on-site in Latin America, we sought to demonstrate the versatility of the protocol for CHIKV detection.

Results: Based on two sequence-specific steps, isothermal RNA amplification and toehold switchbased sensors, we demonstrate sensitivity for target RNA sequences well within the clinically relevant range. Using cultured virus, we then show high specificity against a panel of several arboviruses (DENV 1-4, YFV, ZIKV, CHIKV and MAYV) and similar sensitivity when compared to RT-qPCR for the ZIKV detection, and a diagnostic accuracy of $98.5 \%$ with 268 patient samples. Finally, demonstrating programmability of the approach, we find similar diagnostic performance when the platform is applied to the CHIKV diagnosis. This work, on-site in Latin America, was made possible by an open source lab-ina-box hardware, which provides de-centralized, high-capacity optical measurement.

Conclusion: Here we report one of the first field trials for a synthetic biology-based diagnostic using patient samples. This robust combination (paper-based sensors and lab-in-a-box) have a great potential for use in low-resource areas, particularly in developing countries where ZIKV is now endemic. Our lowcost platform it realizes the goal of large-scale on-site screening for ZIKV infection and to increase the diagnostic capacity of ZIKV-affected low-resource countries.

Keywords: Diagnostic; Zika; Chikungunya 